\title{
Attachment Theory: Progress and Future Directions
}

\author{
R. M. Pasco Fearon \\ University College London
}

Glenn I. Roisman

University of Minnesota

Address for correspondence:

R. M. Pasco Fearon

Research Department of Clinical, Educational and Health Psychology

University College London

Gower Street

London WC1E 6BT

Tel: $+44-207-679-1244$

Email: p.fearon@ucl.ac.uk 


\begin{abstract}
Attachment is a key sub-field in the area of parenting and parent-child relationships research. In this brief overview, we summarise what we consider to be the state-of-the-art of attachment research, focusing primarily on the nature and significance of attachment in infancy and early childhood. We review 4 major topics that are central issues in the scientific literature on attachment: 1) the role of the environment in the development of attachment, 2) the intergenerational transmission of patterns of attachment, 3) the stability of attachment patterns through early adulthood, and 4) the role of attachment in adjustment and maladjustment. We conclude by highlighting several critical unresolved issues and priorities for future research.
\end{abstract}




\section{Introduction}

Attachment is a key topic in the field of parenting and parent child relationships. Originally explicated by Bowlby [1], attachment refers to the tendency of young children to seek contact with one or more consistent caregivers when frightened, worried, or vulnerable, and find such contact comforting. Bowlby's theorising drew heavily on evolutionary theory, ethology, and cybernetics, and conceived of these behaviours as having arisen through natural selection, to maximise survival and reproduction. In that sense, the original theory was not a theory of parenting, but a psychobiological and evolutionary account (indeed, arguably the first) of the nature and function of the child's bond to his or her caregivers. It was primarily the later work of Ainsworth, who identified striking individual differences in attachment behaviour in the now-famous Strange Situation Procedure, that brought a focus on parenting [2]. We now identify four consistent patterns of attachment that can be observed during the Strange Situation in normative and at-risk populations of infants or young children throughout the world: Secure, Insecure-Avoidant, Insecure-Resistant and InsecureDisorganized. Ainsworth's intensive home observations in Uganda and subsequently in the US led her to propose that variation in parenting, and specifically in a particular facet of parenting she labelled sensitivity, was crucial in determining whether a child developed a secure or an insecure attachment relationship with the caregiver. Since that seminal work, attachment research has focused on a number of key questions:

1. Does the evidence support the idea that attachment variation in early life is caused by the environment, not the child's genes, and by sensitivity in particular? 
2. Are patterns of attachment passed from one generation to the next (from parent to child)?

3. Are patterns of attachment carried forward from infancy to adulthood?

4. Are patterns of attachment linked to, and causal in, differences in children's socio-emotional development and adjustment?

In this review, we outline what we consider to be the current state of the field on these 4 key questions. However, we begin by reviewing some critical issues in the measurement of attachment constructs, because, we believe, ongoing limitations in measurement place significant constraints on the ability of research thus far to provide rigorous answers to these 4 questions.

\section{Measurement of attachment}

In recent years, questions have emerged about the validity of the standard view of individual differences in infant attachment security originally formulated by Ainsworth and her colleagues, largely as a result of taxometric and factor analyses conducted by Fraley and Spieker [3] based on the NICHD Study of Early Child Care and Youth Development (SECCYD), one of the largest studies of the Strange Situation conducted to date. Fraley and Spieker discovered from their analyses that, instead of being categorically distributed, variation in attachment security appears compatible with a model in which individual differences in attachment are distributed continuously along two weakly correlated dimensions - one of attachment-related avoidance and another of attachment-related resistance (the latter a combination of resistance and disorganization indicators). Such findings challenge the traditional conceptualization of attachment variation, in which it is implied that avoidance and resistance are: (a) mutually exclusive insecure organizations of 
attachment behavior and (b) categorically distributed in the population. Nonetheless, these findings, which have been extended to measures of adult attachment as well [4, 5], require further exploration and replication in other large sample studies. Moreover, a notable limitation of taxometric and factor analyses in this area is the reliance on rating scales whose individual validity and sensitivity have received relatively scant attention. One particularly critical issue is whether the overlap currently observed in the dimensional framework between resistance and disorganization should be taken as an indication of their common underlying meaning, or a limitation in the way the two constructs are measured. Further work directly contrasting these differing measurement approaches in terms of their distinct or common outcomes or antecedents is thus an important area for future research.

\section{Causes}

Attachment theory makes the bold claim that the causes of variation in attachment security are largely if not entirely environmental, and that caregivers' sensitivity to infants' attachment cues and communications is the primary environmental determinant. In recent years the role of the environment - versus genes - has been tested using the twin method, which provides a powerful test not only of the predicted pre-eminence of the environment but also of the particular importance of the shared environment, a further clear prediction emanating from attachment theory. Remarkably, despite the fact that the majority of domains of development do not show evidence of shared environmental influence, attachment appears to be a striking exception - three independent twin studies, using differing but validated measures, have found significant and substantial shared environmental influence on attachment in infants and young children, and limited evidence of genetic influence [6-8]. Two of these studies also found that the common environmental influences on attachment correlated $[8,9]$, in line with theory, with common variability in maternal sensitivity. 
Crucially, it has also become clear that findings to date relating candidate genes (such as DRD4, 5HTTLPR) to attachment security or disorganization have tended not to replicate and are probably false positives $[10,11]$.

Thus far then, data from twin studies provide relatively good evidence for the proposition that attachment variation is driven predominantly by environmental causes [12]. However, there are three key caveats to this. First, existing twin studies have lacked power to detect genetic effects on sub-types of insecurity_-disorganization being perhaps the most important. Second, no genome-wide association studies with adequate power have been conducted thus far, but one interesting (though underpowered) study found did find at least hints of novel genes that might be relevant to disorganization [13]. So, some (likely small) genetic effects cannot be ruled out. Third, although many studies have replicated the association between attachment and sensitivity, it remains a weak predictor of security, suggesting that there is much we still need to learn about the environmental determinants of attachment. Several factors might account for the weak association [11], including a) measurement error, b) poor ecological validity of existing parenting assessments, c) the wrong parental behaviours being targeted and d) third variables moderating the impact of sensitivity (e.g., genetic susceptibility, see Belsky \& van IJzendoorn, this issue) [14] on attachment or altering the parental behaviours most operative in one context versus another. One critical barrier to achieving greater understanding of the determinants of attachment is that we lack understanding of the precise mechanisms that drive the development of attachment behaviour, such as the inputs the attachment system is sensitive to and the learning mechanisms involved. The evidence that sensitivity-focused interventions can increase rates of secure attachment in RCTs [15-18] (see Dozier \& Barnard, this issue [19]), and that such trials are more effective when they successfully improve sensitivity [15], 
certainly supports the notion that sensitivity is the right ballpark for the hunt for causal mechanisms, but work still needs to be done.

There is a final caveat: the genetic studies discussed above, and indeed the majority of observational studies of attachment and sensitivity, have focused on very young children. We cannot assume that the environmental determinants of attachment in older children are the same. Indeed, a recent twin study of attachment in teenagers, using the well-validated Child Attachment Interview [20], found strong evidence of genetic influence on attachment security, and no evidence of shared environment [21]. The study raises the intriguing possibility that the balance of genetic and environmental influences might shift over the course of development in favour of genetics and non-shared environment- a phenomenon, were it confirmed, that would not be unique to the attachment field [22, 23].

\section{Intergenerational transmission}

The discovery that patterns of infant attachment behaviour might be predictable from the organization of narrative responses observed during the Adult Attachment Interview [24] provided the first evidence that attachment might be transmitted, by what are assumed to be environmental mechanisms, from one generation to the next $[25,26]$, as proposed by Bowlby [27]. An early meta-analysis of 18 studies conducted 20 years ago found the correspondence between parental and infant attachment patterns to be remarkably strong $(r$ $=.47)[28]$. Since then a large number of replications and extensions have been undertaken (95 studies in total), which motivated a recent effort to synthesise and re-evaluate the accumulated evidence since 1995 [29]. This study produced a number of striking findings. First, from 1995 to 2016 the effect size for the inter-generational association has dropped substantially, to an overall $r=.31$. This smaller effect nevertheless remains significant and is 
by no means trivial in size. Second, more than half of these studies were unpublished, and these showed systematically smaller effects (on average $r=.25$ ). Nevertheless, even amongst these 'file drawer' studies, the association was significant, despite the fact that few if any of them — individually — had sufficient power to detect an effect of that size. Also, even though effect sizes have clearly declined over time, the average effect in the most recent decade was still non-trivial in magnitude and significant $(r=.26)$. Thus, despite the smaller overall effect size (which is arguably more realistic, given issues related to measurement error and the likely multifactorial causes of attachment), the wealth of accumulated research suggests that intergenerational transmission of attachment (at least as a correlational, as opposed to causal, phenomenon) is comparatively well supported by the evidence. How the transmission actually works remains relatively poorly understood. Certainly, evidence indicates that variations in parental sensitivity are part of the story, but just as the original 1995 metaanalysis suggested, the gap between parental and infant attachment that cannot be filled by standard measures of sensitivity remains sizeable. Some of the limitations in the measurement of sensitivity that we already alluded to above may be responsible.

\section{Continuity in attachment over time}

The appealing premise of attachment theory that the quality of attachments might be relatively stable across the life course has been of significant interest in the field for many years. Nonetheless, the first longitudinal studies of stability and change in attachment security from infancy into the early years of maturity that emerged around the turn of the century had initially produced notably mixed evidence, with some studies finding substantial stability in attachment security from infancy to adulthood, while others did not. A recent meta-analysis of these generally small sample studies $(r=.14)$ [30], along with the recent completion of an age 18 year AAI assessment of the SECCYD that included over 850 
participants $(r=.12)[31]$, suggest that while attachment security may be significantly stable over the early life course, the magnitude of such stability is weak by conventional standards. Two important caveats are nonetheless necessary here. First, findings from the SECCYD suggest that variation in adult attachment security is more strongly predicted by direct observations of the quality of the early caregiving environment (e.g., maternal sensitivity in childhood) than by measures of attachment taken in infancy [32]. In other words, it is a mistake to conclude that security in adulthood is only weakly associated with childhood experiences with primary caregivers broadly construed. Second, though the most precise estimates available suggest that attachment security is not especially stable across the first two decades of life, when discontinuity exists, it can be explained, at least partly, by attachment-relevant changes in the caregiving environment [33]. For example, in the SECCYD, study participants who remained secure between early childhood and age 18 years (compared with those who changed from secure to insecure) experienced lower levels and a greater decline in maternal sensitivity, were less likely to be living with their fathers, and their mothers reported a larger increase in negative life events in the intervening years.

\section{Attachment and children's socio-emotional adjustment}

Since its inception, attachment theory has been more than a theory of infant behaviour, and a key proposition flowing from it is that security of attachment affects later socio-emotional development. Many authors have argued, with reference to a range of mechanisms - particularly the internal working model (IWM) construct - that early attachment experiences shape how children interpret and respond to social experiences in later life, which in turns impacts on children's social and emotional functioning. A large corpus of research has sought to test this idea by measuring attachment security and relating it to children's peer relationships and adjustment, often over significant periods of time. 
Several linked meta-analytic studies focusing on childhood outcomes have summarized the evidence arising from these studies [34-37]. The findings provide some important indications of the scope and limits of the potential impact of attachment on socio-emotional development. First, the accumulated evidence indicates that attachment security is more strongly correlated with later social competence and externalizing behaviour problems (average $r=.18$ and .15 respectively) $[35,36]$ than internalizing problems $(r=.08$, see Figure 1) [34, 37]. Given the assumed importance of attachment in regulating children's feelings of anxiety or fear, these results may be surprising. Having said that, a natural interpretation of the IWMs hypothesis is that social relationships would be most closely linked to attachment, and the evidence seems broadly consistent with that, particularly if one assumes that externalizing problems in childhood often reflect difficulties with peers. Another possibility of course is that internalizing problems, particularly in young children, are less reliably captured by the measures used in these studies (which often rely on parent report). A further striking finding was that the effects of attachment did not decline as children got older - associations remained the same or even increased when outcomes were measured later in childhood, regardless of the gap in time since attachment was measured (which varied widely). Finally, for both externalizing problems and social competence, the type of outcome assessment seemed to make a difference, with more objective measures yielding larger effects than maternal reports for externalizing outcomes $(r \mathrm{~s} .20-.30$, compared to $r=.11$ ) and assessments not involving close friends similarly yielding larger effects than those with close friends for social competence ( $r$ s of .15-.26, compared to .05) $[35,36]$. Thus, the evidence broadly supports the idea that secure attachment is associated with better socio-emotional outcomes, at least in childhood, but also points to the role of measurement issues, the lack of large main effects, and of some specificity in the insecure subtypes associated with different outcomes. A crucial limitation of all the evidence 
considered in this section is the dearth of experimental studies or cross-lagged longitudinal studies that could test the causal role of attachment in these outcomes. Much more work of this nature is needed.

\section{Concluding remarks}

We have sought to succinctly summarize the state-of-the-science on several important topics on attachment, and we showed that the evidence supports many of the primary hypotheses of attachment theory but also underlines a number of key issues where the evidence indicates a more restricted scope of the effects of attachment than previously assumed, or highlights the limitations of current measurement tools or the typical research designs used. We end by posing some further outstanding questions that future research will need to address:

1. Can we develop better measures of attachment that allow direct assessment of the relationship between indicators, error and underlying constructs? What impact might proper disattenuation of error have on empirical tests of attachment theory?

2. Can tools be developed that allow reliable and consistent measurement over time?

3. Are attachment effects 'just' due to parenting, and if so what does that mean for the importance of attachment per se?

4. Should we be thinking of attachment as a primarily psychological phenomenon, or a biological one? Can a greater use of animal models improve our understanding of human attachment?

5. What is the relationship between normative attachment constructs (secure-base behaviour, security, avoidance, resistance, disorganization) and disorders of, or 
related to, attachment (such as Reactive Attachment Disorder and Disinhibited Social Engagement Disorder, see Zeanah, Humphreys, Fox \& Nelson, [38] this issue).

6. Can we identify the key ingredients of effective attachment interventions, and could such trials be used more effectively to leverage our understanding of the interactive processes involved in the development of attachment?

7. Does attachment mediate the effects of intervention on socio-emotional outcomes? Few trials have been used to test whether attachment is a causal factor in later child adjustment and this is a critical issue for the field.

\section{References}

1. Bowlby J. Attachment and Loss, Vol. 1: Attachment. London: Hogarth Press and the Institute of Psycho-Analysis 1969.

2. Ainsworth MS, Blehar MC, Waters E, et al. Patterns of attachment: A psychological study of the strange situation. Hillsdale, NJ, Lawrence Erlbaum 1978.

3. Fraley RC, Spieker SJ. Are infant attachment patterns continuously or categorically distributed? A taxometric analysis of strange situation behavior. Developmental Psychology 2003;39(3):387-404.

4. Roisman GI, Fraley RC, Belsky J. A taxometric study of the Adult Attachment Interview. Developmental Psychology 2007;43(3):675-86.

5. Fraley RC, Roisman GI. Categories or Dimensions? A Taxometric Analysis of the Adult Attachment Interview. Monographs of the Society for Research in Child Development 2014;79(3):36-50.

6. O'Connor TG, Croft CM. A twin study of attachment in preschool children. Child Development 2001;72(5):1501-11. 
7. Bokhorst CL, Bakermans-Kranenburg MJ, Fearon R, et al. The importance of shared environment in mother-infant attachment security: A behavioral genetic study. Child Development 2003;74(6):1769-82.

8. Roisman GI, Fraley RC. A behavior-genetic study of parenting quality, infant attachment security, and their covariation in a nationally representative sample. Developmental Psychology 2008;44(3):831-39.

9. Fearon R, van IJzendoorn MH, Fonagy P, et al. In search of shared and nonshared environmental factors in security of attachment: A behavior-genetic study of the association between sensitivity and attachment security. Developmental Psychology 2006;42(6):1026-40.

**10. Luijk MP, Roisman GI, Haltigan JD, et al. Dopaminergic, serotonergic, and oxytonergic candidate genes associated with infant attachment security and disorganization? In search of main and interaction effects. Journal of Child Psychology and Psychiatry 2011;52(12):1295-307.

This important study combined two large cohorts that had data on attachment, parenting and a range of candidate genes. Rigorous testing of main effects of single genes, and their interaction with sensitivity if parenting yielded no consistent associations with attachment security or disorganization.

11. Fearon RMP, Belsky J. Precursors of attachment. In: Cassidy J, Shaver PR, eds. Handbook of Attachment: Theory, Research and Clinical Applications. 3rd ed. New York: The Guilford Press 2016:291-313.

12. Bakermans- Kranenburg MJ, van Ijzendoorn MH. Attachment, Parenting, and Genetics. In: Cassidy J, Shaver PR, eds. Handbook of Attachment: Theory, Reseach and Clinical Applications. 3rd ed. New York: The Guilford Press 2016. 
13. Pappa I, Szekely E, Mileva-Seitz VR, et al. Beyond the usual suspects: a multidimensional genetic exploration of infant attachment disorganization and security. Attachment \& Human Development 2015;17(3):288-301.

\section{Belsky \& van IJzendoorn, this issue}

**15. Bakermans-Kranenburg MJ, van IJzendoorn MH, Juffer F. Less is more: Metaanalyses of sensitivity and attachment interventions in early childhood. Psychological Bulletin 2003;129(2):195-215.

This meta-analytic study compiled data from a large number of trials that had attempted to improve attachment security, either through promoting sensitivity, or by working with parents' attachment representations and history, or both. The study made several key points. Firstly, these trials in general were quite effective in improving sensitivity (d $=.33)$ and moderately effective in increasing rates of security $(\mathrm{d}=.20)$. Crucially, studies that focused exclusively on improving sensitivity, and those that succesfully improved sensitivity, had larger effects on security $(d=.39$ and $d=.45$ respectively) than those that did not. Finally, and rather surprisingly, the authors found that studies that employed briefer interventions tended to be more effective $(d=.27)$ in improving rates of security than those that used more intensive ones $(\mathrm{d}=.13-.18)$. The implication was that short and highly targeted interventions focused on improving sensitivity are preferable.

16. Bakermans-Kranenburg MJ, van IJzendoorn MH, Juffer F. Disorganized Infant Attachment and Preventive Interventions: A Review and Meta-Analysis. Infant Mental Health Journal 2005;26(3):191-216. 
17. Bernard K, Dozier M, Bick J, et al. Enhancing attachment organization among maltreated children: Results of a randomized clinical trial. Child Development 2012;83(2):62336.

18. van den Boom DC. The influence of temperament and mothering on attachment and exploration: An experimental manipulation of sensitive responsiveness among lowerclass mothers with irritable infants. Child Development 1994;65(5):1457-77.

19. Dozier and Bernard, this issue

20. Shmueli-Goetz Y, Target M, Fonagy P, et al. The Child Attachment Interview: A psychometric study of reliability and discriminant validity. Developmental Psychology 2008;44(4):939-56.

**21. Fearon P, Shmueli-Goetz Y, Viding E, et al. Genetic and environmental influences on adolescent attachment. Journal of Child Psychology and Psychiatry, and Allied Disciplines 2014;55(9):1033-41.

This study represents the largest twin study of attachment $(\mathrm{N}=551$ twin pairs) using traditional and well-validated instruments conducted to date. The authors used the Child Attachment Interview to assess attachment security and narrative coherence among teenagers aged 15 years. The findings revealed that monozygotic twins were substantially more similar to each other for security and coherence than dyzygotic twins (e.g., the correlations between twins for coherence were $r=.42$ for $\mathrm{MZ}$ twins and $\mathrm{r}=.20$ for DZ twins). Approximately $40 \%$ of the variance in security was estimated to be heritable, and there was no evidence of shared environmental influence, in marked contrast to previous studies with young children. The results suggest that attachment may become increasingly influenced by genes as children grow up. 
22. Briley DA, Tucker-Drob EM. Explaining the Increasing Heritability of Cognitive Ability Across Development A Meta-Analysis of Longitudinal Twin and Adoption Studies. Psychological Science 2013;24(9):1704-13.

23. Bergen SE, Gardner CO, Kendler KS. A meta-analysis of age-related changes in heritability of behavioral phenotypes over adolescence and young adulthood. Behavior Genetics 2007;37(6):738-39.

24. Main M, Goldwyn R. Adult attachment rating and classification systems. In: M.Main, ed. A Typology of Human Attachment Organization Assessed in Discourse, Drawings and Interviews. New York: Cambridge University Press 1990.

25. Fonagy P, Steele H, Steele M. Associations among attachment classifications of mothers, fathers, and their infants: Evidence for a relationship-specific perspective. Child Development 1996;67:541-55.

26. Benoit D, Parker K. Stability and transmission of attachment across three generations. Child Dev 1994;65:1444-57.

27. Bowlby J. Attachment and Loss, Vol. 3: Loss: Sadness and Depression. London: Hogarth Press and Institute of Psycho-Analysis 1980.

28. van IJzendoorn MH. Adult attachment representations, parental responsiveness, and infant attachment: A meta-analysis on the predictive validity of the Adult Attachment Interview. Psychol Bull 1995;117:387-403.

**29. Verhage ML, Schuengel C, Madigan S, et al. Narrowing the Transmission Gap: A Synthesis of Three Decades of Research on Intergenerational Transmission of Attachment. Psychol Bull 2016;142(4):337-66.

This recent meta-analysis drew together more than 30 years' worth of research that had looked at the cross-generational transmission of attachment - comparing parental attachment measured using the AAI, and relating it to infant attachment in the Strange 
Situation and related procedures. The study updated an earlier meta-analysis by van IJzendoorn [28], and was now able to pool nearly 5 times as many samples as the original meta-analysis. The authors tested whether the evidence still favours the hypothesis that attachment patterns are similar (and hence may be transmitted) in parents and children. The results of the analysis confirm that this was indeed the case, but the strength of the association was substantially weaker than observed in 1995 . Interestingly, the effect size could be seen to be declining over time, but nevertheless remain significant and not trivial in size in the most recent studies. The authors also found that studies that focused on clinical or at-risk groups consistently produced weaker associations, which arguably makes sense because the associated risk factors (e.g., postnatal depression), and their impact on parenting, may 'take over' in influencing the child's attachment.

**30. Pinquart M, Feusner C, Ahnert L. Meta-analytic evidence for stability in attachments from infancy to early adulthood. Attachment \& Human Development 2013;15(2):189218.

This meta-analytic study provides quite a comprehensive overview of data examining the short and long-term stability of attachment. It highlighted that short-term stability can be relatively high, but long-term stability is quite weak. It also indicated that 'stability' was lower when comparingn different instruments - e.g., comparing separationreunion procedures like the Strange Situation with representational measures like the AAI.

**31. Groh AM, Roisman GI, Booth-LaForce C, et al. IV. Stability of attachment security from infancy to late adolescence. Monogr Soc Res Child Dev 2014;79(3):51-66.

This is the largest study to examine the long-term stability in attachment from infancy (using the Strange Situation) until, in this case, late adolescence (using the AAI). The 
authors found significant but very modest stability from infancy to late adolescence. However, they also found that broader indicators of the quality of childhood experiences over an extended period of time was quite predictive of adolescent security, which may suggest that adolescent attachment is a multifactorial outcome of a range of earlier developmental processes, and should not be thought of as just the same construct as infancy attachment measured later.

32. Haydon KC, Roisman GI, Owen MT, et al. Shared and Distinctive Antecedents of Adult Attachment Interview State-of-Mind and Inferred-Experience Dimensions. Monographs of the Society for Research in Child Development 2014;79(3):108-25.

33. Booth-LaForce C, Groh AM, Burchinal MR, et al. V. Caregiving and contextual sources of continuity and change in attachment security from infancy to late adolescence. Monogr Soc Res Child Dev 2014;79(3):67-84.

**34. Groh AM, Roisman GI, van IJzendoorn MH, et al. The significance of insecure and disorganized attachment for children's internalizing symptoms: A meta-analytic study. Child Development 2012;83(2):591-610.

**35. Fearon R, Bakermans-Kranenburg MJ, van IJzendoorn MH, et al. The significance of insecure attachment and disorganization in the development of children's externalizing behavior: A meta-analytic study. Child Development 2010;81(2):43556.

**36. Groh AM, Fearon RP, Bakermans-Kranenburg MJ, et al. The significance of attachment security for children's social competence with peers: a meta-analytic study. Attachment \& Human Development 2014;16(2):103-36.

The above three meta-analysies $[34,35,36]$ represent a programmatic attempt to synthesize research into the short-and long-term childhood outcomes associated with early attachment security and insecurity. In each case there was a sizeable and mixed 
literature, making it difficult to summarize the consistent tendencies in a narrative review. Meta-analysis can instead systematically analyse the results of all these studies together and test their overall direction and robustness. The papers suggested that attachment security is associated with better later outcomes, and these effects were quite persistent, seemingly, over time. The association was considerably weaker for outcomes in the internalizing domain than in the externalizing and social competence domains. Furthermore, there was specificity in the sub-types of attachment that stood out in each domain: for externalizing problems, the disorganized children were most at-risk; for internalizing problems it was the avoidant children; for social competence all three insecure categories were at risk to a similar extent.

37. Madigan S, Atkinson L, Laurin K, et al. Attachment and Internalizing Behavior in Early Childhood: A Meta-Analysis. Developmental Psychology 2013;49(4):672-89. 
Figure 1 Average meta-analytic effects sizes relating security and insecurity of attachment to outcome across domains

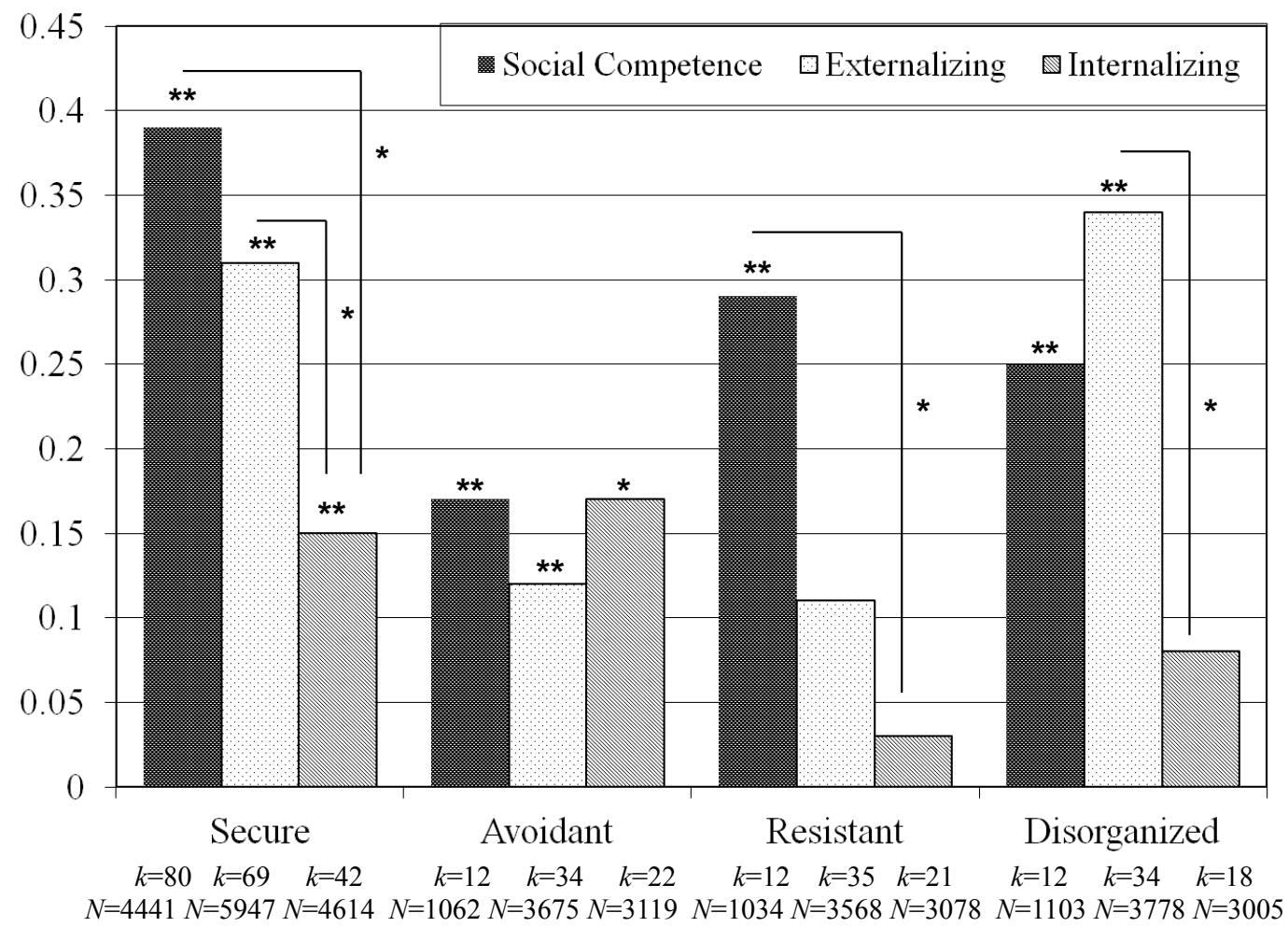

Note: Secure represents the contrast between all reported insecure categories and the secure category (insecure classifications were associated with more difficulties in all domains); the other insecure contrasts compare their respective classifications with all other reported classifications (e.g., avoidant versus secure, resistant and disorganized). 